\title{
Urine Annexin A1 as an Index for Glomerular Injury in Patients
}

\author{
Shuk-Man Ka, ${ }^{1}$ Pei-Yi Tsai, ${ }^{2}$ Tai-Kuang Chao, ${ }^{3}$ Shun-Min Yang, ${ }^{3}$ Yi-Jen Hung, \\ Jin-Shuen Chen, ${ }^{5}$ Hao-Ai Shui, ${ }^{6}$ and Ann Chen $^{3}$ \\ ${ }^{1}$ Graduate Institute of Aerospace and Undersea Medicine, National Defense Medical Center, Taipei, Taiwan \\ ${ }^{2}$ Department of Animal Pharmacology, Development Center for Biotechnology, Taipei, Taiwan \\ ${ }^{3}$ Department of Pathology, Tri-Service General Hospital, National Defense Medical Center, Taipei, Taiwan \\ ${ }^{4}$ Division of Endocrinology \& Metabolism, Department of Internal Medicine, Tri-Service General Hospital, \\ National Defense Medical Center, Taipei, Taiwan \\ ${ }^{5}$ Division of Nephrology, Department of Internal Medicine, Tri-Service General Hospital, National Defense Medical Center, \\ Taipei, Taiwan \\ ${ }^{6}$ Graduate Institute of Medical Sciences, National Defense Medical Center, Taipei, Taiwan
}

Correspondence should be addressed to Ann Chen; annchen31717@gmail.com

Received 27 June 2013; Accepted 20 October 2013; Published 20 January 2014

Academic Editor: Benoit Dugue

Copyright (C) 2014 Shuk-Man Ka et al. This is an open access article distributed under the Creative Commons Attribution License, which permits unrestricted use, distribution, and reproduction in any medium, provided the original work is properly cited.

Background. We recently demonstrated high urine levels of annexin A1 (ANXA1) protein in a mouse Adriamycin-induced glomerulopathy (ADG) model. Objective. To establish ANXA1 as a potential biomarker for glomerular injury in patients. Methods. A time-course study in the mouse ADG model, followed by renal tissues and urine samples from patients with various types of glomerular disorders for ANXA1. Results. Urinary ANXA1 protein was (1) detectable in both the ADG model and in patients except those with minimal change disease (MCD); (2) positively correlated with renal lesions in patients; and (3) early detectable in diabetes patients with normoalbuminuria. Conclusions. ANXA1 is a universal biomarker that is helpful in the early diagnosis, prognostic prediction, and outcome monitoring of glomerular injury. Measurement of urinary ANXA1 protein levels can help in differentiating MCD from other types of glomerular disorders.

\section{Introduction}

Despite their well-known limitations, currently, the most widely used biomarkers for the early detection of chronic kidney disease or acute kidney injury are proteinuria, serum creatinine (Cr), and blood urea nitrogen (BUN). However, all of these indexes are less than optimal and are probably more relevant to later stages of injury, when therapeutic responses might be poor. The value of using serum Cr or albuminuria as a reliable urinary marker is being increasingly challenged in terms of its prediction value in certain renal conditions $[1,2]$. In addition, regardless of the type of glomerular disorder, neither albumin nor $\mathrm{Cr}$ is produced in the kidney. Recently, there has been great interest in strategies aimed at identifying novel biomarkers that can be easily detected in a noninvasive way (such as the use of urine samples) and can predict renal damage during the earlier stages [3]. Glomerular disorders represent a major health care problem because of their high mortality and morbidity rates [4]. Several studies have demonstrated that glomerular disorders are a multifactorial process caused by immune and nonimmune mechanisms that lead to glomerulosclerosis, tubulointerstitial fibrosis, inflammatory infiltration, loss of renal parenchyma, and renal vascular changes $[5,6]$. Although glomerular disorders can cause significant morbidity and mortality, they are treatable and constitute a preventable cause of renal failure and cardiovascular risk [7]. Importantly, the early recognition of the disease and the timely institution of appropriate treatment for patients with glomerular disorders should be beneficial.

We recently demonstrated overproduction of annexin A1 (ANXA1) in the urine in a mouse Adriamycin-induced glomerulopathy (ADG) model, featuring both chronic glomerulosclerosis and tubulointerstitial damage, by using the two-dimensional electrophoresis gel and matrix-assisted 
laser desorption ionization/time of flight mass spectrometry analysis [8]. We also identified another member of the annexin family, annexin A2 (ANXA2), as an early sensor of tubular injury that persists throughout the recovery process of tubular cells in acute renal failure [9] and is upregulated in both the glomerulus and renal tubules in crescentic glomerulonephritis [10]. Annexins are a family of calciumdependent phospholipid-binding proteins that bind reversibly to membranes through calcium-binding loops in their highly conserved core domains $[11,12]$. ANXA1 acts as an important endogenous anti-inflammatory molecule [13-15] and has a variety of other biological activities, including regulation of cell proliferation and cell death signaling and promoting efficient phagocytosis of apoptotic cells [13]. ANXA2 is also involved in a broad range of biological processes, such as cell migration [16], plasminogen activation [17], signal transduction [18], and resolution of inflammation [19]. Furthermore, ANXA1 or ANXA2 mRNA or protein levels are altered in various human diseases, for example, cystic fibrosis $[20,21]$, hepatocellular carcinoma $[22,23]$, prostate carcinoma [24-26], pancreatic cancer [27, 28], breast cancer [29$31]$, and renal cell carcinoma $[32,33]$. However, the expression patterns and potential roles of ANXA1 and ANXA2 remain unclear in most glomerular disorders.

In the present study, we characterized the expression pattern and tissue distribution of ANXA1 and ANXA2 in the ADG model over time and confirmed these results in a large number of different types of human glomerular disorders. We infer that ANXA1 and ANXA2 may play important pathogenic roles in glomerular disorders. Importantly, only ANXA1, which is significantly increased in the kidney and urine, can be used as an early noninvasive urinary biomarker for most types of glomerular disorders.

\section{Methods}

2.1. ADG Animal Model and Experimental Protocol. ADG was induced in C57BL/6 mice by a single injection of Adriamycin as described previously [34]. Urine was collected using metabolic cages, and proteinuria and BUN and Cr levels in the serum were evaluated as described previously [35]. Mice were sacrificed at days $0,3,7,14$, and 21 for pathological evaluation, extraction of mRNA for real-time reverse transcription-polymerase chain reaction (RT-PCR) analysis, and detection of urinary levels of specific proteins. All animal experiments were performed with the ethical approval of the Institutional Animal Care and Use Committee of the National Defense Medical Center, Taiwan, and according to the ethical rules in the NIH Guide for the Care and Use of Laboratory Animals.

\subsection{Renal Tissues and Urine Samples from Patients with} Glomerular Disorders. Eighty-one patients with glomerular disorders, consisting of minimal change disease (MCD) $(n=$ 19), IgA nephropathy (IgAN) $(n=10)$, membranous glomerulonephritis (MGN) $(n=12)$, focal segmental glomerulosclerosis (FSGS) $(n=11)$, crescentic glomerulonephritis $(\mathrm{CrGN})(n=5)$, diabetic nephropathy $(\mathrm{DN})(n=8)$, or lupus nephritis $(\mathrm{LN})(n=16)$, were retrospectively included in this study. Renal biopsy specimens and urine samples were obtained at the time of diagnosis. Renal tissues taken from the unaffected pole of kidneys removed because of renal cell carcinoma were used as normal controls. Normal urine samples were collected from apparently healthy volunteers ( $n=15$ ). The urinary albumin excretion is examined by the ratio of urinary albumin concentration to urinary $\mathrm{Cr}$ concentration (UACR) [36].

On the other hand, 47 urine samples from diabetic patients without albuminuria (normoalbuminuria) or with early kidney involvement (microalbuminuria or macroalbuminuria) were also included in this study. In those patients, normoalbuminuria was defined as UACR $<30 \mathrm{mg} / \mathrm{g}$, microalbuminuria was defined as UACR cutoff of $30-299 \mathrm{mg} / \mathrm{g}$, and macroalbuminuria was defined as UACR $\geq 300$ according to the criteria of the American Diabetes Association [37]. Detailed patient characteristics are given in Supplementary Table 1 in Supplementary Material available online at http:// dx.doi.org/10.1155/2014/854163.

The patients were followed at the Tri-Service General Hospital, Taipei, Taiwan, and all patients who contributed samples signed an informed consent form, as required by the regulations of the Institutional Review Board of the TriService General Hospital, National Defense Medical Center, Taipei, Taiwan.

2.3. Renal Pathological Evaluation. Renal tissues were fixed in $10 \%$ buffered formalin, embedded in paraffin, and stained with hematoxylin and eosin for routine histopathologic evaluation. For mice histopathologic evaluations of sclerosis, at least 50 glomeruli in renal tissue sections for each case were examined. Severity of sclerosis was semiquantified by morphological changes on a scale of 1-4, as described previously [38]. For patients, pathological parameters such as activity indices and chronicity indices were approached by renal pathologists as described previously [39] involving semiquantitative scoring of specific biopsy features. The proportion was calculated for the following four major components: (1) glomerular proliferation, (2) glomerular sclerosis, (3) interstitial inflammation, (4) interstitial fibrosis, and (5) crescentic formation, respectively. Differences in scoring between the pathologists were resolved by rereviewing the biopsies and thus reaching a consensus.

2.4. Real-Time RT-PCR Examination. Renal cortex RNA was extracted using TRIzol reagent (Invitrogen, CA, USA) according to the manufacturer's instructions and real-time RT-PCR was used to verify altered gene expression in the ADG animal model as described previously [34]. The primers used are shown as follows: mouse ANXA1: $5^{\prime}$-GGGACTTGGAACAGATGAAGAC-3', 5' -GTCTGTCCCCTTTCTTCCTTTCT-3'; mouse ANXA2: $5^{\prime}$-GTGGATGAGGTCACCATTGTC-3', $5^{\prime}$-GTCGGTTCCTTTCCTCTTCAC-3'; and mouse GAPDH: $5^{\prime}$-TCCGCCCCTTCTGCCGATG$3^{\prime}, 5^{\prime}$-CACGGAAGGCCATGCCAGTGA-3'. Amplifications were normalized to GAPDH using the $2^{-\Delta \Delta \mathrm{CT}}$ method [40]. 
2.5. In Situ Hybridization (ISH). ISH was performed as described previously [10]. Formalin-fixed and paraffinembedded renal sections were used. The cDNAs used as templates for the synthesis of the specific RNA probes were generated from mouse kidney or human renal biopsies by RT-PCR. The primers used for mice as described in the real-time RT-PCR section and the primers used for human were ANXA1: $5^{\prime}$-TGAGGAGGTTGTTTTAGCTCTG- ${ }^{\prime}, 5^{\prime}$ GTTCTTGATGCCAAAATCTCAA- $3^{\prime}$; ANXA2: $5^{\prime}$-TCTACTGTTCACGAAATCCTGT- ${ }^{\prime}$, 5 $^{\prime}$-TTCAATAGGCCAAAATCACCG-3'. Scoring of mRNA expression levels in kidney tissues was performed under light microscopy and 50 randomly selected cortical fields were examined in each section as described previously [10].

2.6. Immunohistochemistry (IHC). Formalin-fixed and paraffin-embedded renal sections were prepared as described previously [41] and were stained using primary antibodies against mouse ANXA1 (Zymed Laboratories, CA, USA), human ANXA1 (BD Biosciences, CA, USA), or human/ mouse ANXA2 (Santa Cruz Biotechnology, CA, USA), biotinylated secondary antibody, streptavidin-conjugated horseradish peroxidase, and AEC reagent (DakoCytomation, CA, USA). Semiquantitative evaluation was performed as described above for ISH.

2.7. Western Blot Analysis of Urine Samples. Protein levels of ANXA1 and ANXA2 in urine samples from mice with ADG or patients were measured by Western blot analysis as described previously [10] using the antibodies described in the IHC section. The data are presented as the ratio of the density of the target protein band to the Cr concentration in the urine as described previously [10].

2.8. Data Analysis. The results are presented as the mean \pm SEM or median. Comparisons between two groups were performed using Student's $t$-test. The correlation coefficient was Pearson's $r$ values. A $P$ value $<0.05$ was considered statistically significant.

\section{Results}

3.1. Clinical Manifestations and Pathological Features of the $A D G$ Model. Persistent heavy proteinuria and deteriorated renal function were observed in the ADG mice after a single Adriamycin injection. Compared to normal controls, the mice showed an increase in Cr-corrected urine protein levels $(\mathrm{mg} / \mathrm{mg})$ on day $7(1.14 \pm 0.11$ versus $0.28 \pm 0.09, P<0.05)$, and levels remained high till the end of the experiment on day $21(2.21 \pm 0.27, P<0.005)$. BUN levels in the serum showed a significant and progressive increase from day 14 $(63.2 \pm 8.12 \mathrm{mg} / \mathrm{dL}, P<0.01)$ to day $21(96.81 \pm 5.42 \mathrm{mg} / \mathrm{dL}$, $P<0.005)$ compared to normal controls $(23.9 \pm 2.34 \mathrm{mg} / \mathrm{dL})$, and serum Cr levels also showed a significant increase from day $7(0.96 \pm 0.09 \mathrm{mg} / \mathrm{dL}$ versus $0.46 \pm 0.04 \mathrm{mg} / \mathrm{dL}, P<0.05)$ and progressively increased until the mice were sacrificed on day $21(2.45 \pm 0.18 \mathrm{mg} / \mathrm{dL}, P<0.005)$.
Histopathological examination was performed on kidney sections at different time points (Figure 1). Expansion of the extracellular matrix and deposition of hyaline mass in the glomeruli were occasionally seen on day 7 and became obvious and statistically significant from day 14 to day 21 . Importantly, the mice exhibited a significant and steady increase in the percentage of glomeruli containing epithelial hyperplastic lesions from day 14 to day 21 , suggesting a progressive pathological status. The sclerosis score is shown in Figure 1(f) and correlated well with the severity of the renal biological parameters.

3.2. Increased $A N X A 1$ and $A N X A 2 m R N A$ Levels in the $A D G$ Model. To determine whether ANXA1 and ANXA2 mRNA levels changed with disease evolution, real-time RT-PCR analysis was performed on total RNA extracted from the renal cortex at various points after ADG induction. As shown in Figure 2(a), renal ANXA1 mRNA levels were significantly increased in the ADG model as early as day 3 and remained persistently increased up to day 21 when the mice were sacrificed (day $3, P<0.01$; day $7, P<0.05$; day $14, P<0.01$; day $21, P<0.005)$ compared to day 0 . In contrast, as shown in Figure 2(b), ANXA2 mRNA levels only showed a significant increase on day 14 and day 21 compared to day $0(P<0.005$ for both).

Next, we used ISH to determine which cells in the renal tissues showed ANXA1 and ANXA2 gene expression over time. As shown in Figure 3, ANXA1 mRNA was expressed as early as day 3 (Figures 3(b) and 3(f)), predominantly in the podocytes and some renal tubules, consistent with the real-time RT-PCR findings. ANXA1 staining of the parietal epithelial cells, podocytes, and epithelial hyperplastic lesions of the glomerulus was seen from day 7 until day 21, when the mice were sacrificed, although few renal tubules were positive (Figures 3(c)-3(f)). However, only basal levels of ANXA2 mRNA were seen in the renal tubules from day 0 to day 3 (Figures 3(g), 3(h), 3(l)), with a gradual increase on day 7 (Figures 3(i) and 3(l)). On days 14 and 21 (Figures 3(j)-3(l)), the pattern of ANXA2 mRNA expression was similar to that of ANXA1 mRNA, except that ANXA2 mRNA levels were even higher than those for ANXA1 in certain renal tubules with potential regeneration activity.

3.3. Increased ANXA1 and ANXA2 Protein Expression in the $A D G$ Model. The tissue and cell location of ANXA1 and ANXA2 protein expression in the kidney were evaluated by IHC. Both ANXA1 (Figures 4(a)-4(f)) and ANXA2 (Figures $4(\mathrm{~g})-4(\mathrm{l}))$ were significantly increased as early as day 7 and till day 21 , compared to day 0 . Moreover, approximately $25 \%$ of the ANXA1 stained cells showed a nuclear staining pattern in addition to the cytoplasmic staining pattern.

3.4. Dynamic Changes in Urine ANXA1 Levels in the ADG Model. We examined whether these proteins were excreted in the urine using Western blot analysis. As shown in Figures 5(a) and 5(b), ANXA1 protein was not detectable in the urine on days 0 and 3 , but a significant increase was seen from day 7 to day 21 (day 7, $672.73 \pm 121.06 \mathrm{U} / \mathrm{mg} \mathrm{Cr}$; day 14, 


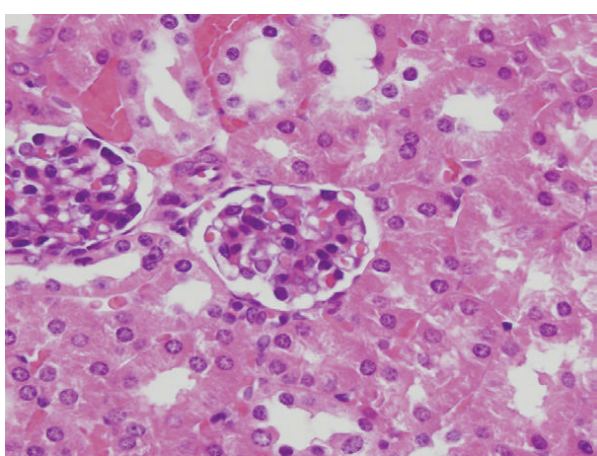

(a)

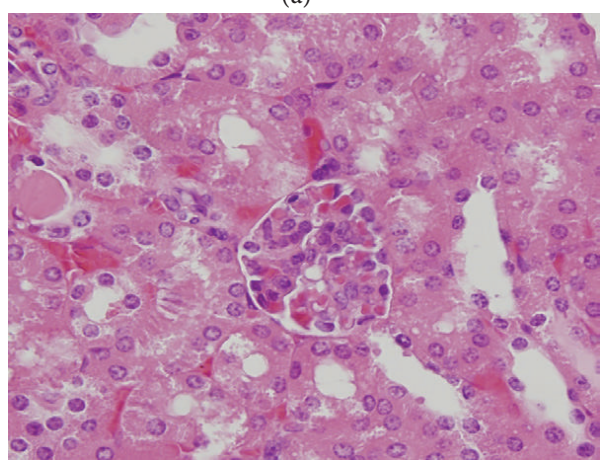

(c)

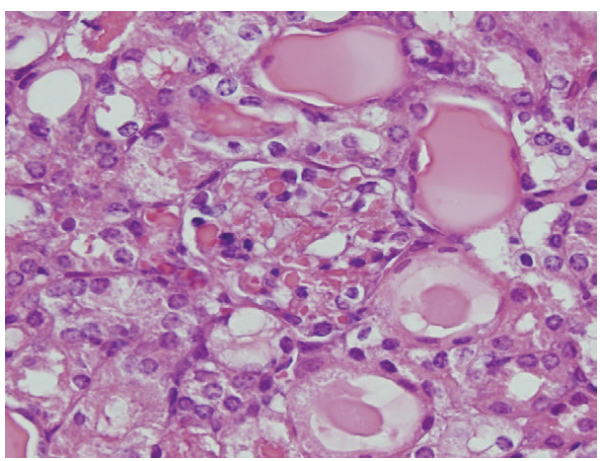

(e)

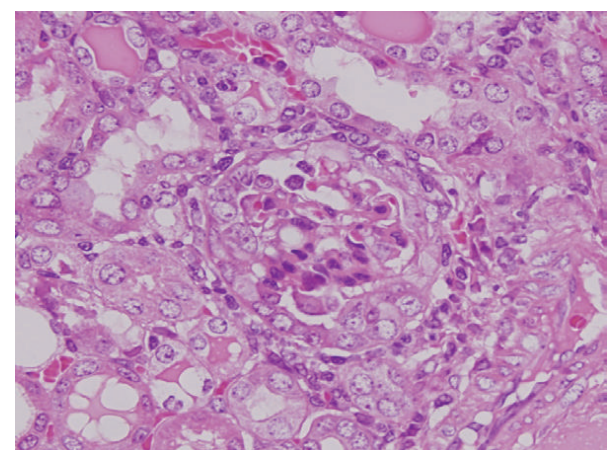

(b)

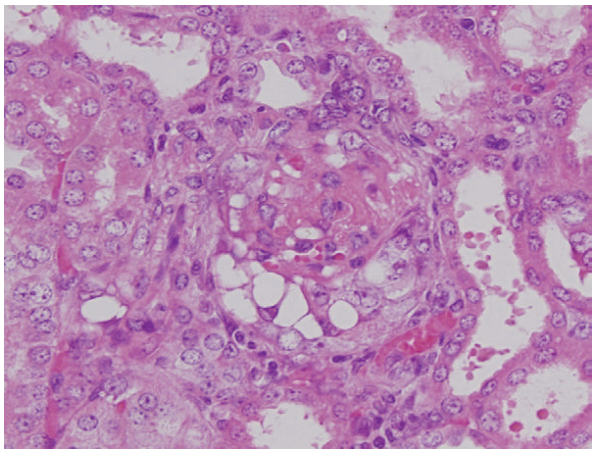

(d)

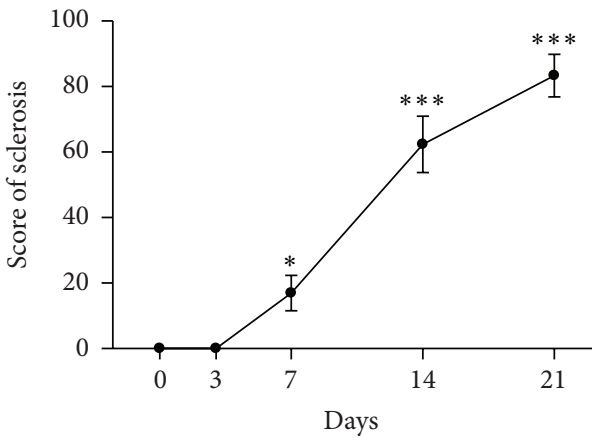

(f)

Figure 1: Glomerular histopathology in ADG mice. (a)-(e) Kidney tissue histopathological sections on day 0 (a), day 3 (b), day 7 (c), day 14 (d), or day 21 (e), showing a gradual increase in sclerosis in the glomeruli (hematoxylin and eosin staining). Original magnification, $\times 400$. (f) Semiquantitative plot showing the sclerosis scores for the glomeruli in the tissue sections. Each point represents the mean \pm SEM for six mice per group. ${ }^{*} P<0.05,{ }^{* * *} P<0.005$ compared to the normal controls (day 0 ).

887.27 \pm 97.65 U/mg Cr; day 21, $1232.44 \pm 197.88 \mathrm{U} / \mathrm{mg} \mathrm{Cr}$ versus day $0,0 \mathrm{U} / \mathrm{mg}$ ) (all $P<0.005$ ). Importantly, both 37 $\mathrm{kDa}$ intact ANXA1 and $33 \mathrm{kDa}$ cleaved ANXA1 were observed in the urine (Figure 5(a)). In contrast, ANXA2 was not detected in the urine in normal control mice or at any time in the ADG model.

3.5. Enhanced ANXA1 and ANXA2 mRNA and Protein Expression in Renal Tissues of Patients with Various Glomerular Disorders except MCD. We then examined ANXA1 and ANXA2 mRNA and protein expression in patients with various types of glomerular disorders by ISH or IHC staining.
As shown in Figure 6, although ISH showed that very little ANXA1 mRNA was expressed in the tubules or glomeruli in the normal controls (bottom line, left panels) and patients with MCD (top line, left panels), most biopsy tissues from other types of glomerular disorders were found to have high levels of ANXA1 mRNA in the glomeruli examined. The major sites of ANXA1 mRNA expression were in the podocytes and parietal epithelial cells in IgAN, MGN, and FSGS. Dense intracellular staining was seen in the fibrotic region of glomeruli, especially in the crescentic formation in CrGN, DN, and LN. In the tubulointerstitial compartment, ANXA1 mRNA expression was mainly focally localized in regenerating tubules and infiltrating inflammatory cells. ANXA2 


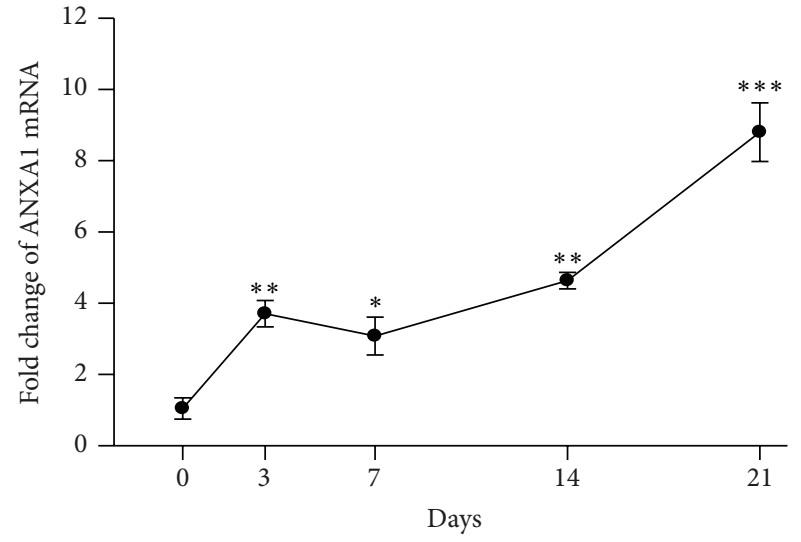

(a)

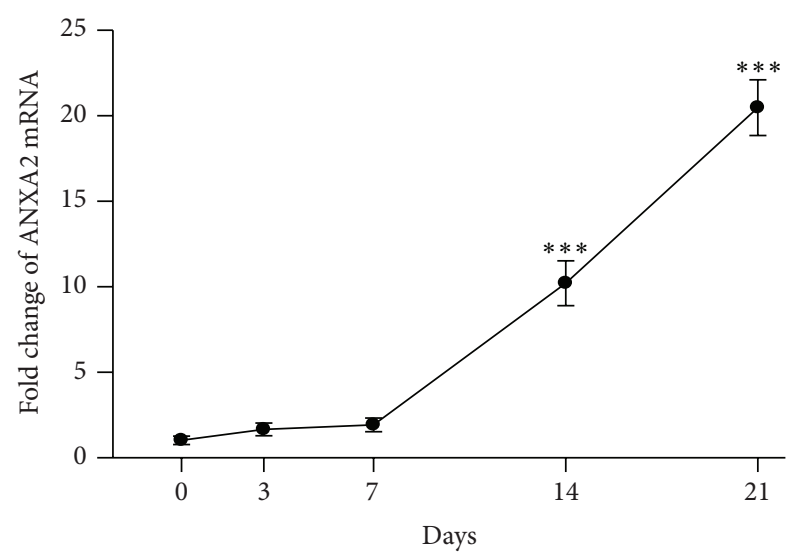

(b)

FIGURE 2: Quantitative analysis of mRNA levels in renal cortex from ADG mice by real-time RT-PCR over time. (a) ANXA1 and (b) ANXA2. Each point represents the mean \pm SEM for six mice per group. ${ }^{*} P<0.05,{ }^{* *} P<0.01,{ }^{* * *} P<0.005$ compared to the controls (day 0 ).

mRNA showed a similar distribution and intensity to that of ANXA1 mRNA.

The pattern of ANXA1 and ANXA2 protein expression, as demonstrated by IHC, was generally similar to that of the mRNAs. Moreover, consistent with the results in the ADG model, approximately 25\% of ANXA1-stained cells showed nuclear staining in addition to cytoplasmic staining, and ANXA2 protein was also expressed in the plasma membrane in epithelial cells in the crescentic formation.

\subsection{Increased Urinary ANXA1 Protein Levels in Patients}

3.6.1. In Various Glomerular Disorders except MCD. To determine whether ANXA1 could serve as a biomarker for noninvasive diagnosis and prognostic prediction, we measured urine protein levels in patients with various types of glomerular disorders and apparently healthy donors by Western blot analysis. As shown in Table 1, ANXA1 protein was detectable in the urine of almost all patients, compared to normal controls, except those with MCD (less than $10.52 \%$ of cases were detected). Importantly, excluding MCD, urinary levels of ANXA1 were significantly positively correlated with the levels of UACR $(r=0.43, P<0.001)$.

3.6.2. Positive Correlation with Renal Lesions. We then attempted to identify the relationship of urinary ANXA1 levels to renal pathological lesions. We classified the patients' specimens as group 1: primary GN: nonproliferative $\mathrm{GN}$, including MCD, MGN, and FSGS; group 2: primary GN: proliferative GN, including IgAN and $\mathrm{CrGN}$, and group 3: secondary GN, including DN and LN, as described previously $[42,43]$. As shown in Figure 7, urine ANXA1 levels were significantly increased in these three groups compared to normal control (all $P<0.005$ ). Moreover, urinary ANXA1 was significantly increased in group $3(773.13 \pm 144.96 \mathrm{U} / \mathrm{mg}$ $\mathrm{Cr})$ compared to both group $1(217.10 \pm 40.62 \mathrm{U} / \mathrm{mg} \mathrm{Cr}, P<$ $0.005)$ and group $2(294.01 \pm 71.89 \mathrm{U} / \mathrm{mg} \mathrm{Cr}, P<0.01)$. In all these three groups, urinary ANXA1 levels were significantly
TABLE 1: Urine ANXA1 levels and urinary albumin excretion in patients with various types of glomerular disorder.

\begin{tabular}{lcc}
\hline Diagnosis & $\mathrm{U} / \mathrm{mg} \mathrm{Cr}^{*}$ & $\mathrm{UACR}^{*}(\mathrm{mg} / \mathrm{g})$ \\
\hline MCD & 0 & 172.68 \\
IgAN & 130.87 & 43.43 \\
MGN & 157.62 & 88.97 \\
FSGS & 382.18 & 105.53 \\
CrGN & 524.29 & 267.17 \\
DN & 563.27 & 215.65 \\
LN & 554.59 & 117.01 \\
Normal control & 0 & 2.5 \\
\hline
\end{tabular}

* Data are median.

MCD: minimal change disease; MGN: membranous glomerulonephritis; FSGS: focal segmental glomerulosclerosis; IgAN: IgA nephropathy; CrGN: crescentic glomerulonephritis; DN: diabetic nephropathy; LN: lupus nephritis.

positively correlated with glomerular proliferation (group 1, $r=0.51, P<0.01$; group $2, r=0.75, P<0.005$; group $3, r=0.51, P<0.05$ ) and interstitial inflammation (group $1, r=0.56, P<0.005$; group $2, r=0.63, P<0.05$; group $3, r=0.45, P<0.05$ ) (Table 2 ). In addition, the levels of urinary ANXA1 also correlated well with glomerular sclerosis in group $1(r=0.43, P<0.01)$ and group $3(r=0.58$, $P<0.05)$. Moreover, urinary ANXA1 levels were significantly positively correlated with crescentic formation in group 2 and group 3 (group 2, $r=0.69$; group $3, r=0.73$ ) (both $P<0.05$ ).

Combined all patients with various glomerular disorders, urinary ANXA1 protein levels positively correlated with the score of glomerular proliferation $(r=0.67, P<0.001)$, interstitial inflammation $(r=0.54, P<0.005)$, and glomerular sclerosis $(r=0.44, P<0.001)$.

3.6.3. As an Early Urinary Biomarker for Diabetic Nephropathy. Because diabetes is the most common single cause of end-stage renal disease (ESRD) in the world [44], we 
ANXA1

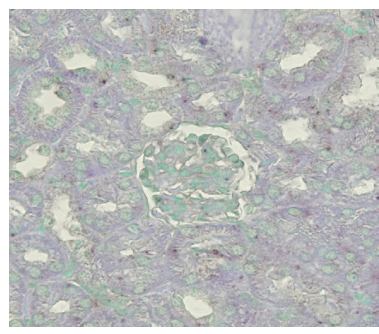

(a)

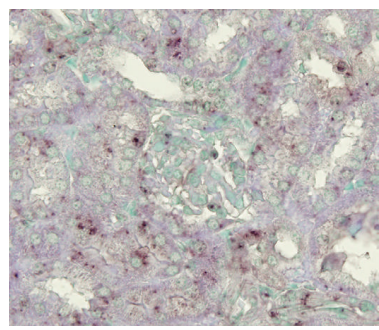

(b)

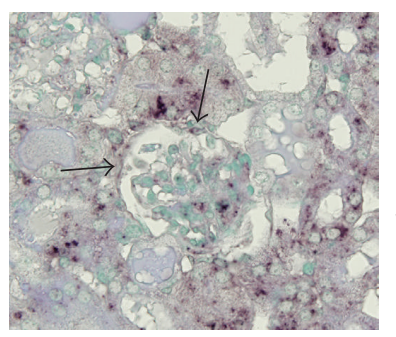

(c)

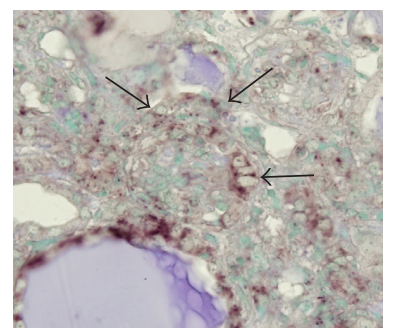

(d)

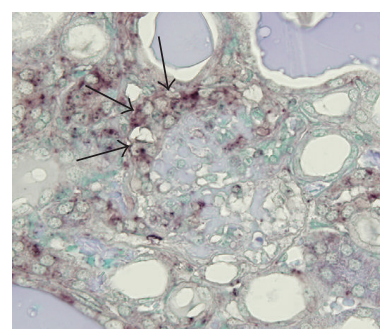

(e)

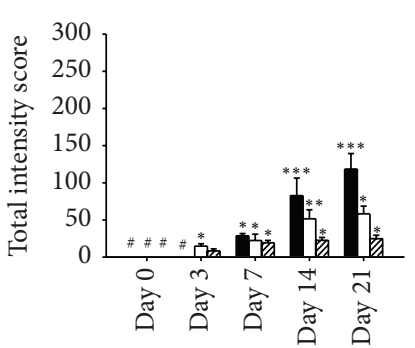

- Parietal epithelial cells $\square$ Podocytes mes Mesangial cells

(f)
ANXA2

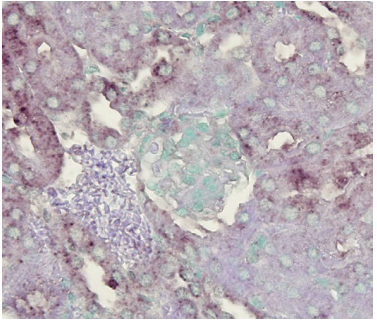

(g)

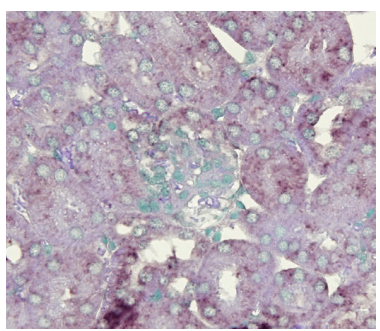

(h)
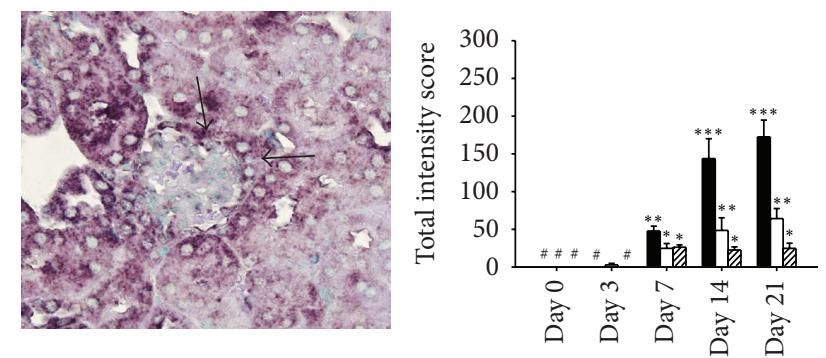

- Parietal epithelial cells $\square$ Podocytes mesangial cells

(i)

Figure 3: Cellular expression of ANXA1 and ANXA2 mRNAs demonstrated by ISH at different time points after ADG induction. (a)-(f) ANXA1 and (g)-(l) ANXA2. Kidney tissue sections on day 0 ((a) and (g)), day 3 ((b) and (h)), day 7 ((c) and (i)), day 14 ((d) and (j)), or day $21((\mathrm{e})$ and $(\mathrm{k}))$. The arrows indicate epithelial cells in the crescent-like formation of the glomerulus. Original magnification, $\times 400$. (f) and (l) Semiquantitative analysis of cellular protein expression by ISH. Scoring was performed for the three major components of the parietal epithelial cells (solid bars), podocytes (open bars), and mesangial cells (hatched bars). Each bar represents the mean \pm SEM for six mice per group. ${ }^{*} P<0.05 ;{ }^{* *} P<0.01 ;{ }^{* * *} P<0.005$, compared to the normal controls (day 0$)$. \#: not detectable.

recruited 47 urine samples from diabetes patients without albuminuria (normoalbuminuria) or with early kidney involvement (microalbuminuria and macroalbuminuria) in this study. As shown in Table 3, the urinary ANXA1 was detectable as early as the stage of normoalbuminuria in diabetes patients with $52.94 \%$, microalbuminuria with $72.73 \%$, and macroalbuminuria with $100 \%$.

\section{Discussion}

These findings support the value of the use of ANXA1 in various glomerular disorders as (1) a universal diagnostic and prognostic biomarker, (2) a noninvasive urinary early marker that can help in the differential diagnosis of the progressive types of glomerular disorders that can cause nephrotic syndrome from MCD, the most common, but favorable, type of glomerular disorder, and (3) a potential index for differentiating systemic diseases with kidney involvement from primary glomerular disorders.

Studies over the past 10 years have shown that the $37 \mathrm{kDa}$ ANXA1 is cleaved at the $\mathrm{N}$-terminus to generate the $33 \mathrm{kDa}$ isoform, an event that occurs following the adhesion of blood neutrophils to the capillary endothelial cells, a process that activates protein externalization [45]. In the present study, both the $37 \mathrm{kDa}$ intact ANXA1 and $33 \mathrm{kDa}$ cleaved ANXA1 were detected in the urine (Figure 5). It has been demonstrated that the full-length ANXA1 and its cleaved form are released by apoptotic polymorphonuclear neutrophils and apoptotic mesangial cells [46]. Our data suggest that urinary ANXA1 comes from damaged and/or apoptotic renal tissues. ANXA1 became detectable in the urine as early as day 7 in the ADG model (Figure 5) and, 
ANXA1

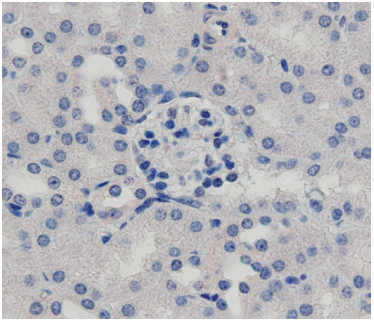

(a)

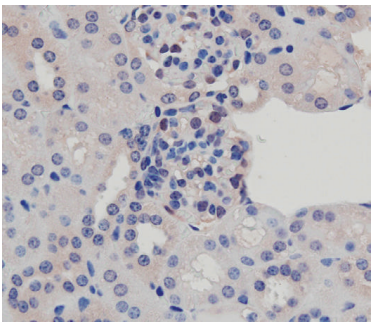

(b)

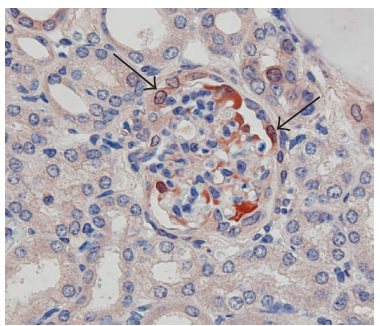

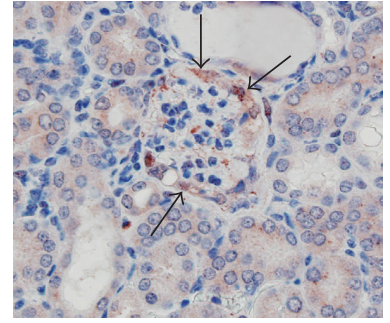

(d)

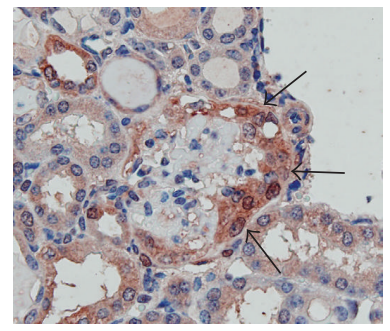

(e)

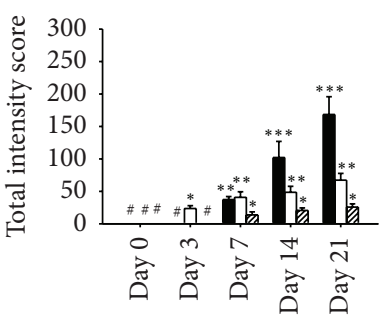

- Parietal epithelial cells $\square$ Podocytes

[mes Mesangial cells

ANXA2

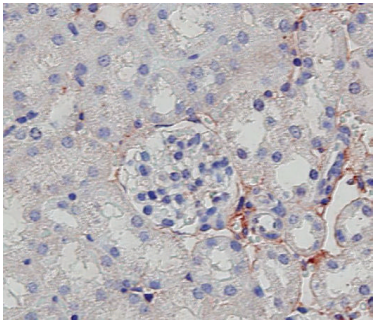

(g)

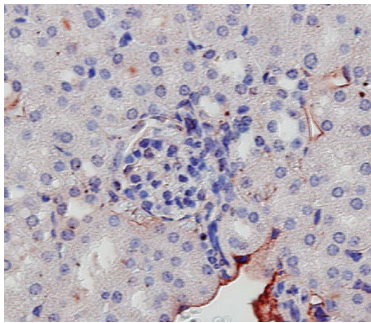

(h)

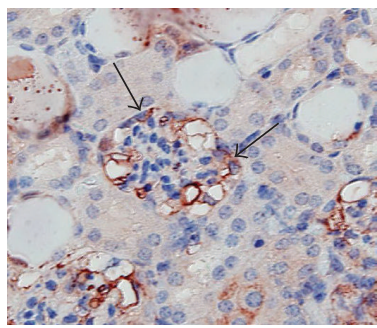

(i)

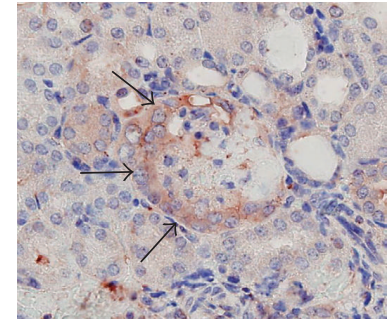

(j)

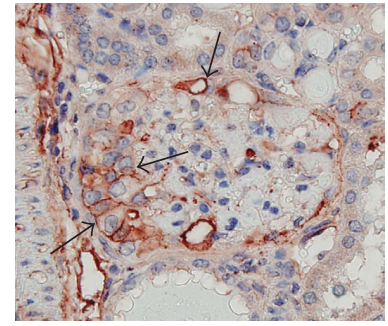

(k)

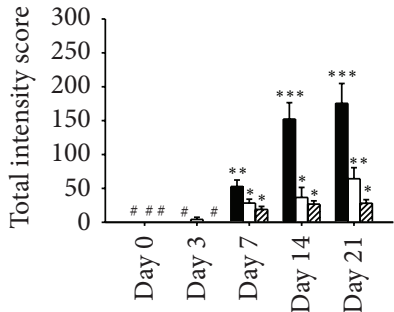

- Parietal epithelial cells $\square$ Podocytes men Mesangial cells

(l)

FIGURE 4: Detection of ANXA1 and ANXA2 protein expression in kidney tissue by IHC at different time points after ADG induction. (a)-(f) ANXA1 and (g)-(l) ANXA2. Kidney tissue sections on day 0 ((a) and (g)), day 3 ((b) and (h)), day 7 ((c) and (i)), day 14 ((d) and (j)), or day $21((\mathrm{e})$ and $(\mathrm{k}))$. The arrows indicate epithelial cells in the crescent-like formation of the glomerulus. Original magnification, $\times 400$. (f) and (l) Semiquantitative analysis of cellular protein expression by IHC. Scoring was performed for the three major components of the parietal epithelial cells (solid bars), podocytes (open bars), and mesangial cells (hatched bars). Each bar represents the mean \pm SEM for six mice per group. ${ }^{*} P<0.05 ;{ }^{* *} P<0.01 ;{ }^{* * *} P<0.005$, compared to the normal controls (day 0 ). \#: not detectable.

importantly, was detectable in almost all urine samples from patients with glomerular disorders except those with MCD (less than $10.52 \%$ of cases were detected). Moreover, urinary levels of ANXA1 in secondary glomerular diseases, such as LN and DN, were significantly higher than those in primary glomerular disorders, including nonproliferative or proliferative GN (Figure 7), suggesting that ANXA1 may be helpful in the differential diagnosis of secondary versus primary glomerular disorders.

In fact, early detection of risk leading to the possibility of intervention before advanced renal injury has occurred is an obviously important goal. We use urine from diabetes patients, the most common single cause of ESRD in the world, to evaluate whether urinary ANXA1 could serve as an early biomarker for kidney injury. At present, microalbuminuria is considered the best available noninvasive marker for diabetes risk, but recently more and more studies have proved that it has inadequate specificity and sensitivity [47, 48]. In this study, we demonstrated that ANXA1 protein was earlier present in $52.94 \%$ of diabetes patients with normoalbuminuria. Thus, we infer that urinary ANXA1 may serve as an early diagnosis marker for glomerular injury.

ANXA1 has been reported to be highly expressed in epithelial cells of Bowman's capsule, macula densa, and medullary/papillary collecting ducts of the normal rat kidney [49] and overexpressed in multiple human cancers, including conventional renal cell carcinomas [33]. In the present study, we first demonstrated that podocytes, epithelial cells in the crescent, and parietal epithelial cells were the major sites of ANXA1 expression in the glomerulus, as well as certain actively regenerating renal tubules (Figures 3, 4, and 6).

Several reports have demonstrated that cytoplasmic ANXA1 can be induced to translocate into the nucleus by treatment with specific stimuli, such as EGF in the case of 
TABLE 2: Correlation of urinary ANXA1 levels with pathological findings in patients with various types of glomerular disorders.

\begin{tabular}{|c|c|c|c|c|c|c|}
\hline \multicolumn{7}{|c|}{ Primary GN } \\
\hline & \multicolumn{2}{|c|}{ Nonproliferative GN } & \multicolumn{2}{|c|}{ Proliferative GN } & \multicolumn{2}{|c|}{ Secondary GN } \\
\hline & $r$ & $P$ & $r$ & $P$ & $r$ & $P$ \\
\hline Glomerular proliferation & 0.51 & 0.008 & 0.75 & 0.004 & 0.51 & 0.043 \\
\hline Glomerular sclerosis & 0.43 & 0.006 & 0.55 & 0.063 & 0.58 & 0.033 \\
\hline Interstitial inflammation & 0.56 & 0.003 & 0.63 & 0.029 & 0.45 & 0.027 \\
\hline Interstitial fibrosis & -0.12 & 0.612 & 0.30 & 0.346 & 0.22 & 0.408 \\
\hline Crescentic formation & - & - & 0.69 & 0.012 & 0.73 & 0.001 \\
\hline
\end{tabular}

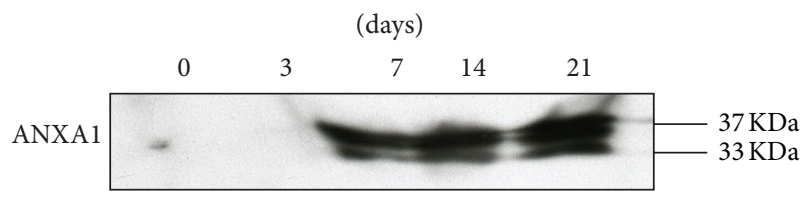

(a)

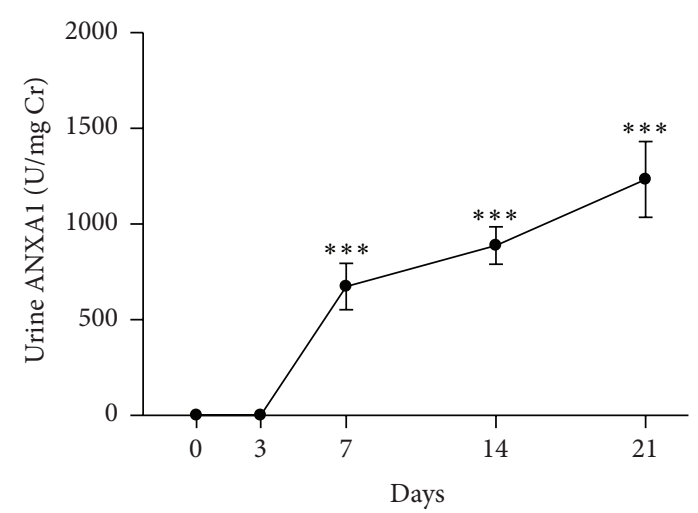

(b)

FIGURE 5: Detection of urine ANXA1 levels by Western blotting during the course of the ADG model. (a) Representative Western blot of urine samples; the molecular weights are shown on the right. (b) Quantitative analysis of the ratio of the protein band density to urinary creatinine over time. Each point represents the mean \pm SEM for six mice per group. ${ }^{* * *} P<0.005$ compared to the normal controls (day 0$)$.

alveolar basal epithelial cells [50] and PMA in the case of human embryonic kidney cells [51], suggesting that ANXA1 nuclear translocation is involved in the regulation of cellular proliferation and may be directly involved in tumor invasion [52]. Although approximately $25 \%$ of ANXA1-positive cells in patients showed nuclear staining in addition to cytoplasmic staining, the significance of nuclear ANXA1 staining remains unclear and deserves further investigation.

We demonstrated here that ANXA1 may be involved in protecting glomerular proliferation and inflammation in glomerular injury. These findings from ISH and IHC data demonstrated that time-dependent enhancement in ANXA1 and the urinary levels of ANXA1 significantly correlate between the degree of glomerular proliferation and renal interstitial inflammation from histopathological assays, suggesting that ANXA1 plays an important inhibition role in
TABLE 3: Urine ANXA1 levels in diabetic patients without albuminuria or with early kidney involvement.

\begin{tabular}{lcc}
\hline & $\mathrm{U} / \mathrm{mg} \mathrm{Cr}^{*}$ & Percentage \\
\hline Normoalbuminuria & 3.17 & $52.94 \%(n=14)$ \\
Microalbuminuria & 4.90 & $72.73 \%(n=11)$ \\
Macroalbuminuria & 19.90 & $100 \%(n=22)$ \\
\hline${ }^{*}$ Data are median. & &
\end{tabular}

renal injury processes. Both proliferation and inflammation are believed to play a major role in the progression of glomerular disorders $[5,53]$. ANXA1 has been demonstrated to inhibit cell proliferation in several cell types, including mesangial cells [54], by inhibition of cyclin D1 expression through ERK1/2 MAPK signaling [55]. In contrast, overexpression of ANXA2 promotes cell proliferation in lung cancers [56] and inhibits apoptosis in breast cancers [57]. In addition, ANXA1 has been described as playing a homeostatic role in cells of the innate immune system $[15,58]$ and to act as a protective and anti-inflammatory protein in models of rheumatoid arthritis and myocardial infarct $[15,59,60]$ by direct inhibition of phospholipase A2 [14]. Besides, ANXA2 can be detected in renal tissues from mice under normal status and renal injury $[9,10]$, and soluble ANXA2 tetramer has been reported to act as a soluble mediator of macrophage activation [19]. Fan et al. [61] demonstrated that ANXA1 and ANXA2 act as bridging molecules linking phagocyte and target cells and promote the phagocytosis of apoptotic lymphocytes, which results in reduction of inflammation. In the ADG model, we showed upregulation of ANXA1 and ANXA2 during the course of the disease, and this effect is consistent with our previous report of increased F4/80+ macrophage infiltration in the kidney in the mouse model [38]. Based on our observations and these published data, it is suggested that the very early enhanced renal expression of ANXA1 mRNA and protein in the ADG model might act to protect against glomerular proliferation and renal inflammation.

McArthur et al. [62] showed that ANXA1 exerts an autocrine-paracrine action leading to Rho kinase (ROCK) activation and actin polymerization. Rho/ROCK signaling has been linked to actin polymerization [63] and is involved in tubulointerstitial fibrosis [64] and glomerulosclerosis [65]. We also demonstrated that ANXA1 positively correlated with the degree of glomerular sclerosis in all patients with various types of glomerular disorders and crescentic formation in 

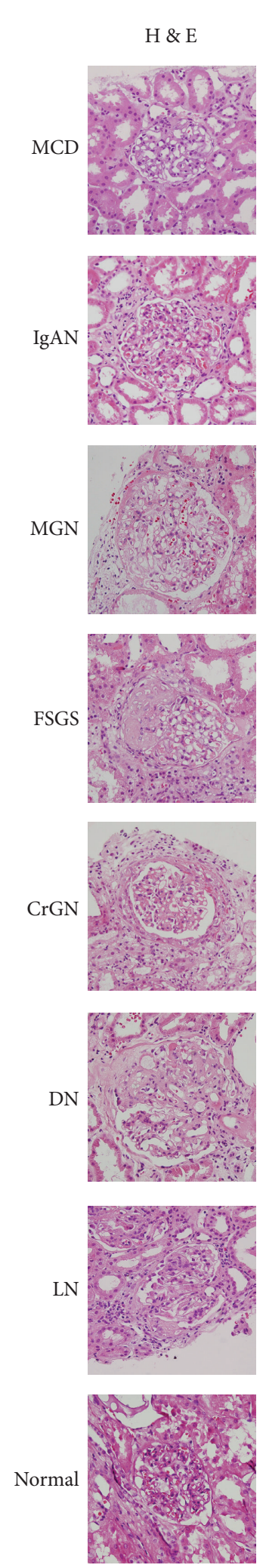
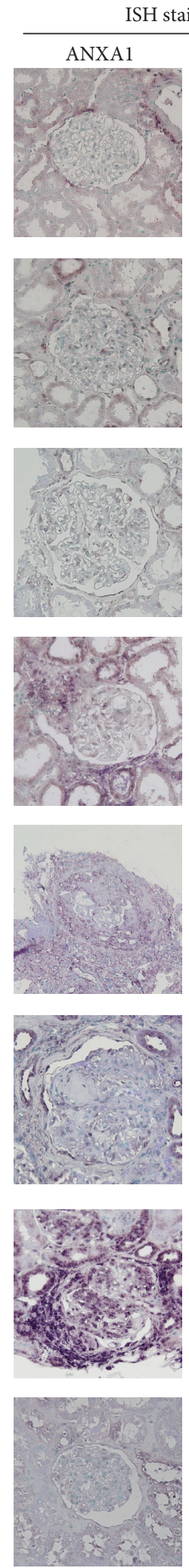
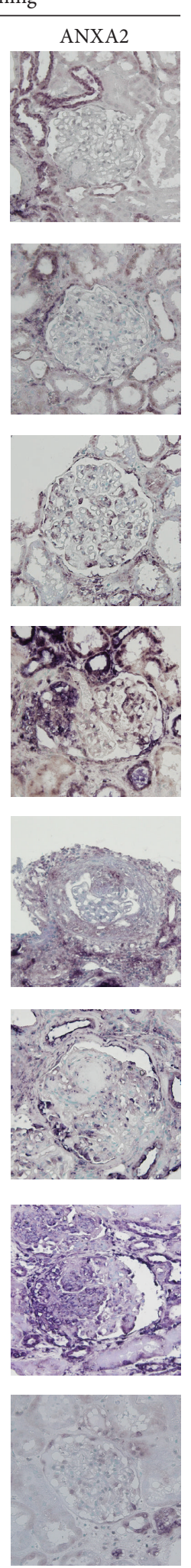
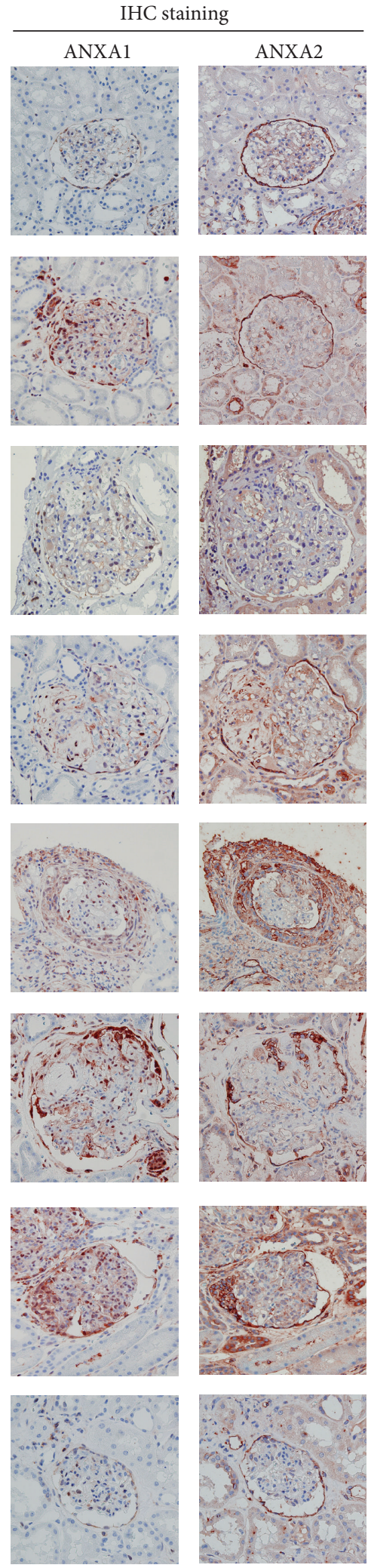

FIGURE 6: Detection of ANXA1 and ANXA2 mRNA and protein expression in kidney tissues from patients with various types of glomerular disorders by ISH or IHC. Representative histopathology of kidney tissues by haematoxylin and eosin staining. Original magnification, $\times 400$. MCD: minimal change disease; IgAN: IgA nephropathy; MGN: membranous glomerulonephritis; FSGS: focal segmental glomerulosclerosis; CrGN: crescentic glomerulonephritis; DN: diabetic nephropathy; LN: lupus nephritis. 


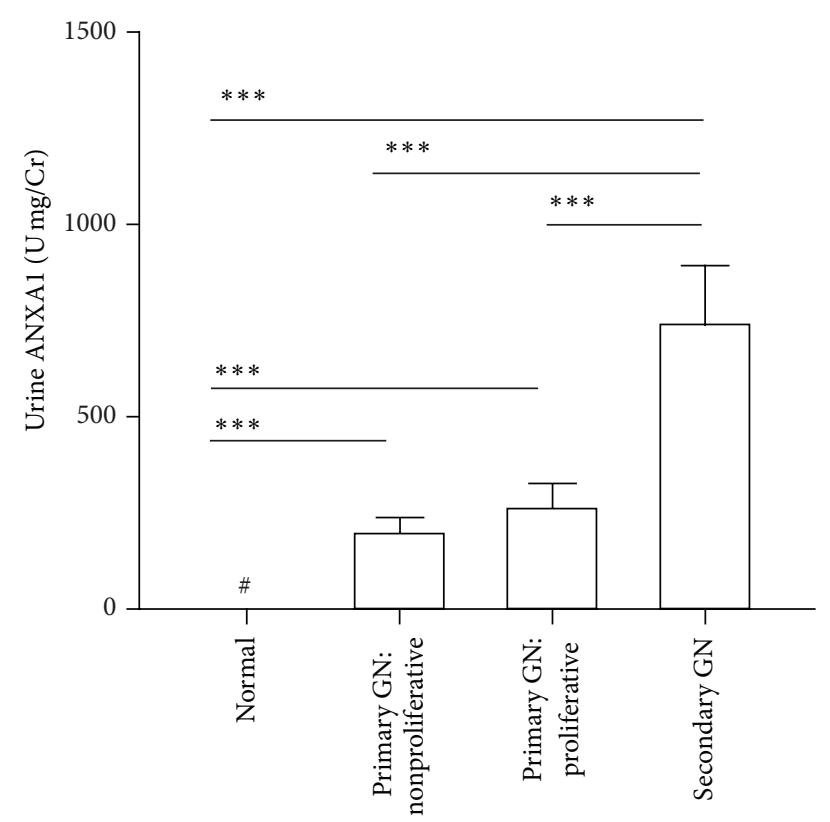

FIGURE 7: Urine ANXA1 levels correlated in patients with various types of glomerular disorders. GN: glomerulonephritis. ${ }^{* * *} P<$ 0.005. \#: not detectable.

patients with primary GN: proliferative GN and secondary GN. Further studies on the roles of ANXA1 in renal fibrosis/sclerosis and in protecting renal tissues from proliferation and inflammation during the evolution of glomerular disorders are warranted. It is necessary to add other new techniques such as flow cytometry or multiplex assays in future investigation to evaluate the ANXA1 expression in the renal samples of patients for pursuing ANXA1 as a reliable biomarker with predictive and diagnostic values.

In summary, we demonstrated that (1) the detection of ANXA1 protein in the urine may be helpful as a noninvasive approach for the diagnosis and prognostic prediction of various glomerular injury; (2) ANXA1 can be used as a sensitive urinary biomarker in a rapid test allowing most forms of glomerular disorders to be differentiated from MCD; (3) ANXA1 shows potential for differentiating between secondary and primary glomerular disorders; and (4) ANXA1 and ANXA2 can serve as injury markers and may play roles in the persistence and progression of glomerular disorders.

\section{Conflict of Interests}

The authors declare that there is no conflict of interests associated with this paper.

\section{Acknowledgments}

This study was supported by grants from the Ministry of Economic Affairs (100-EC-17-A-19-S1-161) and the National Science Council (NSC-101-2321-B-016-003), Taiwan.

\section{References}

[1] A. Ishani, G. A. Grandits, R. H. Grimm et al., "Association of single measurements of dipstick proteinuria, estimated glomerular filtration rate, and hematocrit with 25-year incidence of endstage renal disease in the multiple risk factor intervention trial," Journal of the American Society of Nephrology, vol. 17, no. 5, pp. 1444-1452, 2006.

[2] W. F. Keane, Z. Zhang, P. A. Lyle et al., "Risk scores for predicting outcomes in patients with type 2 diabetes and nephropathy: the RENAAL study," Clinical journal of the American Society of Nephrology, vol. 1, no. 4, pp. 761-767, 2006.

[3] M. H. Rosner, "Urinary biomarkers for the detection of renal injury," Advances in clinical chemistry, vol. 49, pp. 73-97, 2009.

[4] J. R. Timoshanko and P. G. Tipping, "Resident kidney cells and their involvement in glomerulonephritis," Current Drug Targets: Inflammation and Allergy, vol. 4, no. 3, pp. 353-362, 2005.

[5] A. B. Fogo, "Mechanisms of progression of chronic kidney disease," Pediatric Nephrology, vol. 22, no. 12, pp. 2011-2022, 2007.

[6] J. L. Olson and R. H. Heptinstall, "Biology of disease. Nonimmunologic mechanisms of glomerular injury," Laboratory Investigation, vol. 59, no. 5, pp. 564-578, 1988.

[7] P. W. Mathieson, "Glomerulonephritis: is it worth worrying about?” Clinical Medicine, vol. 5, no. 3, pp. 264-266, 2005.

[8] H.-A. Shui, T.-H. Huang, S.-M. Ka, P.-H. Chen, Y.-F. Lin, and A. Chen, "Urinary proteome and potential biomarkers associated with serial pathogenesis steps of focal segmental glomerulosclerosis," Nephrology Dialysis Transplantation, vol. 23, no. 1, pp. 176-185, 2008.

[9] C.-W. Cheng, A. Rifai, K. A. Shuk-Man et al., "Calcium-binding proteins annexin A2 and S100A6 are sensors of tubular injury and recovery in acute renal failure," Kidney International, vol. 68, no. 6, pp. 2694-2703, 2005.

[10] S.-M. Ka, A. Rifai, J.-H. Chen et al., "Glomerular crescentrelated biomarkers in a murine model of chronic graft versus host disease," Nephrology Dialysis Transplantation, vol. 21, no. 2, pp. 288-298, 2006.

[11] V. Gerke and S. E. Moss, "Annexins: from structure to function," Physiological Reviews, vol. 82, no. 2, pp. 331-371, 2002.

[12] A. C. Rintala-Dempsey, A. Rezvanpour, and G. S. Shaw, "S100annexin complexes-structural insights," The FEBS Journal, vol. 275, no. 20, pp. 4956-4966, 2008.

[13] L. H. K. Lim and S. Pervaiz, "Annexin 1: the new face of an old molecule," The FASEB Journal, vol. 21, no. 4, pp. 968-975, 2007.

[14] M. Perretti and R. J. Flower, "Annexin 1 and the biology of the neutrophil," Journal of Leukocyte Biology, vol. 76, no. 1, pp. 2529, 2004.

[15] M. Perretti and F. N. E. Gavins, "Annexin 1: an endogenous antiinflammatory protein," News in Physiological Sciences, vol. 18, no. 2, pp. 60-64, 2003.

[16] L. Tatenhorst, U. Rescher, V. Gerke, and W. Paulus, "Knockdown of annexin 2 decreases migration of human glioma cells in vitro," Neuropathology and Applied Neurobiology, vol. 32, no. 3, pp. 271-277, 2006.

[17] Q. Ling, A. T. Jacovina, A. Deora et al., "Annexin II regulates fibrin homeostasis and neoangiogenesis in vivo," Journal of Clinical Investigation, vol. 113, no. 1, pp. 38-48, 2004.

[18] Y. Biener, R. Feinstein, M. Mayak, Y. Kaburagi, T. Kadowaki, and Y. Zick, "Annexin II is a novel player in insulin signal 
transduction: possible association between annexin II phosphorylation and insulin receptor internalization," Journal of Biological Chemistry, vol. 271, no. 46, pp. 29489-29496, 1996.

[19] J. F. A. Swisher, U. Khatri, and G. M. Feldman, "Annexin A2 is a soluble mediator of macrophage activation," Journal of Leukocyte Biology, vol. 82, no. 5, pp. 1174-1184, 2007.

[20] L. A. Borthwick, J. Mcgaw, G. Conner et al., "The formation of the cAMP/protein kinase A-dependent annexin 2-S100A10 complex with cystic fibrosis conductance regulator protein (CFTR) regulates CFTR channel function," Molecular Biology of the Cell, vol. 18, no. 9, pp. 3388-3397, 2007.

[21] F. H. C. Tsao, K. C. Meyer, X. Chen, N. S. Rosenthal, and J. Hu, "Degradation of annexin I in bronchoalveolar lavage fluid from patients with cystic fibrosis," American Journal of Respiratory Cell and Molecular Biology, vol. 18, no. 1, pp. 120-128, 1998.

[22] T. Masaki, M. Tokuda, M. Ohnishi et al., "Enhanced expression of the protein kinase substrate annexin I in human hepatocellular carcinoma," Hepatology, vol. 24, no. 1, pp. 72-81, 1996.

[23] H. S. Mohammad, K. Kurokohchi, H. Yoneyama et al., "Annexin A2 expression and phosphorylation are up-regulated in hepatocellular carcinoma," International Journal of Oncology, vol. 33, no. 6, pp. 1157-1163, 2008.

[24] C. P. Paweletz, D. K. Ornstein, M. J. Roth et al., "Loss of annexin 1 correlates with early onset of tumorigenesis in esophageal and prostate carcinoma," Cancer Research, vol. 60, no. 22, pp. 6293$6297,2000$.

[25] Y. Shiozawa, A. M. Havens, Y. Jung et al., "Annexin II/annexin II receptor axis regulates adhesion, migration, homing, and growth of prostate cancer," Journal of Cellular Biochemistry, vol. 105, no. 2, pp. 370-380, 2008.

[26] W. Xin, D. R. Rhodes, C. Ingold, A. M. Chinnaiyan, and M. A. Rubin, "Dysregulation of the annexin family protein family is associated with prostate cancer progression," American Journal of Pathology, vol. 162, no. 1, pp. 255-261, 2003.

[27] X.-F. Bai, X.-G. Ni, P. Zhao et al., "Overexpression of annexin 1 in pancreatic cancer and its clinical significance," World Journal of Gastroenterology, vol. 10, no. 10, pp. 1466-1470, 2004.

[28] E. Ortiz-Zapater, S. Peiró, O. Roda et al., “Tissue plasminogen activator induces pancreatic cancer cell proliferation by a noncatalytic mechanism that requires extracellular signal-regulated kinase $1 / 2$ activation through epidermal growth factor receptor and annexin A2," American Journal of Pathology, vol. 170, no. 5, pp. 1573-1584, 2007.

[29] M. R. Sharma, L. Koltowski, R. T. Ownbey, G. P. Tuszynski, and M. C. Sharma, "Angiogenesis-associated protein annexin II in breast cancer: selective expression in invasive breast cancer and contribution to tumor invasion and progression," Experimental and Molecular Pathology, vol. 81, no. 2, pp. 146-156, 2006.

[30] D. Shen, H. R. Chang, Z. Chen et al., "Loss of annexin A1 expression in human breast cancer detected by multiple highthroughput analyses," Biochemical and Biophysical Research Communications, vol. 326, no. 1, pp. 218-227, 2004.

[31] D. Shen, F. Nooraie, Y. Elshimali et al., "Decreased expression of annexin A1 is correlated with breast cancer development and progression as determined by a tissue microarray analysis," Human Pathology, vol. 37, no. 12, pp. 1583-1591, 2006.

[32] T. Domoto, Y. Miyama, H. Suzuki et al., "Evaluation of S100A10, annexin II and B-FABP expression as markers for renal cell carcinoma," Cancer Science, vol. 98, no. 1, pp. 77-82, 2007.

[33] E. Solito, A. Kamal, F. Russo-Marie, J. C. Buckingham, S. Marullo, and M. Perretti, "A novel calcium-dependent proapoptotic effect of annexin 1 on human neutrophils," The FASEB journal, vol. 17, no. 11, pp. 1544-1546, 2003.

[34] P.-Y. Tsai, S.-M. Ka, T.-K. Chao et al., "Antroquinonol reduces oxidative stress by enhancing the Nrf2 signaling pathway and inhibits inflammation and sclerosis in focal segmental glomerulosclerosis mice," Free Radical Biology and Medicine, vol. 50, no. 11, pp. 1503-1516, 2011.

[35] A. Chen, L.-F. Sheu, Y.-S. Ho et al., "Experimental focal segmental glomerulosclerosis in mice," Nephron, vol. 78, no. 4, pp. 440-452, 1998.

[36] S. Romundstad, J. Holmen, K. Kvenild, H. Hallan, and H. Ellekjær, "Microalbuminuria and all-cause mortality in 2,089 apparently healthy individuals: a 4.4-year follow-up study. The Nord-Trøndelag Health Study (HUNT), Norway," American Journal of Kidney Diseases, vol. 42, no. 3, pp. 466-473, 2003.

[37] Expert Committee on the Diagnosis Clasification of Diabetes Mellitus, "American diabetes association: clinical practice recommendations," Diabetes Care, vol. 25, supplement 1, pp. 1-147, 2002.

[38] H.-A. Shui, S.-M. Ka, S.-M. Yang, Y.-F. Lin, Y.-F. Lo, and A. Chen, "Osteopontin as an injury marker expressing in epithelial hyperplasia lesions helpful in prognosis of focal segmental glomerulosclerosis," Translational Research, vol. 150, no. 4, pp. 216-222, 2007.

[39] F. Yu, L.-H. Wu, Y. Tan et al., “Tubulointerstitial lesions of patients with lupus nephritis classified by the 2003 international society of nephrology and renal pathology society system," Kidney International, vol. 77, no. 9, pp. 820-829, 2010.

[40] C.-W. Cheng, S.-M. Ka, S.-M. Yang et al., "Nephronectin expression in nephrotoxic acute tubular necrosis," Nephrology Dialysis Transplantation, vol. 23, no. 1, pp. 101-109, 2008.

[41] H. Y. Lan, X. Q. Yu, N. Yang et al., "De novo glomerular osteopontin expression in rat crescentic glomerulonephritis," Kidney International, vol. 53, no. 1, pp. 136-145, 1998.

[42] G. B. Fogazzi, L. Saglimbeni, G. Banfi et al., "Urinary sediment features in proliferative and non-proliferative glomerular diseases," Journal of Nephrology, vol. 18, no. 6, pp. 703-710, 2005.

[43] R. Minutolo, M. M. Balletta, F. Catapano et al., "Mesangial hypercellularity predicts antiproteinuric response to dual blockade of RAS in primary glomerulonephritis," Kidney International, vol. 70, no. 6, pp. 1170-1176, 2006.

[44] H. Jiang, G. Guan, R. Zhang et al., "Increased urinary excretion of orosomucoid is a risk predictor of diabetic nephropathy," Nephrology, vol. 14, no. 3, pp. 332-337, 2009.

[45] L. Vong, F. D’Acquisto, M. Pederzoli-Ribeil et al., "Annexin 1 cleavage in activated neutrophils: a pivotal role for proteinase 3," Journal of Biological Chemistry, vol. 282, no. 41, pp. 2999830004, 2007.

[46] M. Scannell, M. B. Flanagan, A. DeStefani et al., "Annexin-1 and peptide derivatives are released by apoptotic cells and stimulate phagocytosis of apoptotic neutrophils by macrophages," Journal of Immunology, vol. 178, no. 7, pp. 4595-4605, 2007.

[47] M. L. Caramori, P. Fioretto, and M. Mauer, "The need for early predictors of diabetic nephropathy risk: is albumin excretion rate sufficient?" Diabetes, vol. 49, no. 9, pp. 1399-1408, 2000.

[48] B. P. Tabaei, A. S. Al-Kassab, L. L. Ilag, C. M. Zawacki, and W. H. Herman, "Does microalbuminuria predict diabetic nephropathy?” Diabetes Care, vol. 24, no. 9, pp. 1560-1566, 2001.

[49] A. Markoff and V. Gerke, "Expression and functions of annexins in the kidney," American Journal of Physiology: Renal Physiology, vol. 289, no. 5, pp. F949-F956, 2005. 
[50] S. Radke, J. Austermann, F. Russo-Marie, V. Gerke, and U. Rescher, "Specific association of annexin 1 with plasma membrane-resident and internalized EGF receptors mediated through the protein core domain," The FEBS Letters, vol. 578, no. 1-2, pp. 95-98, 2004.

[51] Y. S. Kim, J. Ko, I. S. Kim et al., "PKC $\delta$-dependent cleavage and nuclear translocation of annexin A1 by phorbol 12-myristate 13acetate," European Journal of Biochemistry, vol. 270, no. 20, pp. 4089-4094, 2003.

[52] C.-Y. Lin, Y.-M. Jeng, H.-Y. Chou et al., "Nuclear localization of annexin $\mathrm{Al}$ is a prognostic factor in oral squamous cell carcinoma," Journal of Surgical Oncology, vol. 97, no. 6, pp. 544$550,2008$.

[53] D. M. Silverstein, "Inflammation in chronic kidney disease:role in the progression of renal and cardiovascular disease," Pediatric Nephrology, vol. 24, no. 8, pp. 1445-1452, 2009.

[54] S. B. Kim, W. S. Yang, S. O. Lee, K. P. Lee, J. S. Park, and D. S. Na, "Lipocortin-1 inhibits proliferation of cultured human mesangial cells," Nephron, vol. 74, no. 1, pp. 39-44, 1996.

[55] L. C. Alldridge and C. E. Bryant, "Annexin 1 regulates cell proliferation by disruption of cell morphology and inhibition of cyclin D1 expression through sustained activation of the ERK1/2 MAPK signal," Experimental Cell Research, vol. 290, no. 1, pp. 93-107, 2003.

[56] Y. Huang, Y. Jin, C.-H. Yan et al., "Involvement of Annexin A2 in p53 induced apoptosis in lung cancer," Molecular and Cellular Biochemistry, vol. 309, no. 1-2, pp. 117-123, 2008.

[57] S. Chuthapisith, B. E. Bean, G. Cowley et al., "Annexins in human breast cancer: possible predictors of pathological response to neoadjuvant chemotherapy," European Journal of Cancer, vol. 45, no. 7, pp. 1274-1281, 2009.

[58] M. Perretti and E. Solito, "Annexin 1 and neutrophil apoptosis," Biochemical Society Transactions, vol. 32, no. 3, pp. 507-510, 2004.

[59] M. La, M. D’Amico, S. Bandiera et al., "Annexin 1 peptides protect against experimental myocardial ischemia-reperfusion: analysis of their mechanism of action," The FASEB Journal, vol. 15, no. 12, pp. 2247-2256, 2001.

[60] Y. Yang, P. Hutchinson, and E. F. Morand, "Inhibitory effect of annexin I on synovial inflammation in rat adjuvant arthritis," Arthritis \& Rheumatism, vol. 42, pp. 1538-1544, 1999.

[61] X. Fan, S. Krahling, D. Smith, P. Williamson, and R. A. Schlegel, "Macrophage surface expression of annexins I and II in the phagocytosis of apoptotic lymphocytes," Molecular Biology of the Cell, vol. 15, no. 6, pp. 2863-2872, 2004.

[62] S. McArthur, S. Yazid, H. Christian et al., "Annexin A1 regulates hormone exocytosis through a mechanism involving actin reorganization," The FASEB Journal, vol. 23, no. 11, pp. 4000-4010, 2009.

[63] R. L. Simões and I. M. Fierro, "Involvement of the Rhokinase/myosin light chain kinase pathway on human monocyte chemotaxis induced by ATL-1, an aspirin-triggered lipoxin A4 synthetic analog," Journal of Immunology, vol. 175, no. 3, pp. 1843-1850, 2005.

[64] K. Nagatoya, T. Moriyama, N. Kawada et al., "Y-27632 prevents tubulointerstitial fibrosis in mouse kidneys with unilateral ureteral obstruction," Kidney International, vol. 61, no. 5, pp. 1684-1695, 2002.

[65] T. Nishikimi and H. Matsuoka, "Molecular mechanisms and therapeutic strategies of chronic renal injury: renoprotective effect of rho-kinase inhibitor in hypertensive glomerulosclerosis," Journal of Pharmacological Sciences, vol. 100, no. 1, pp. 22$28,2006$. 


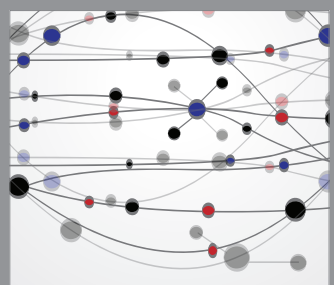

The Scientific World Journal
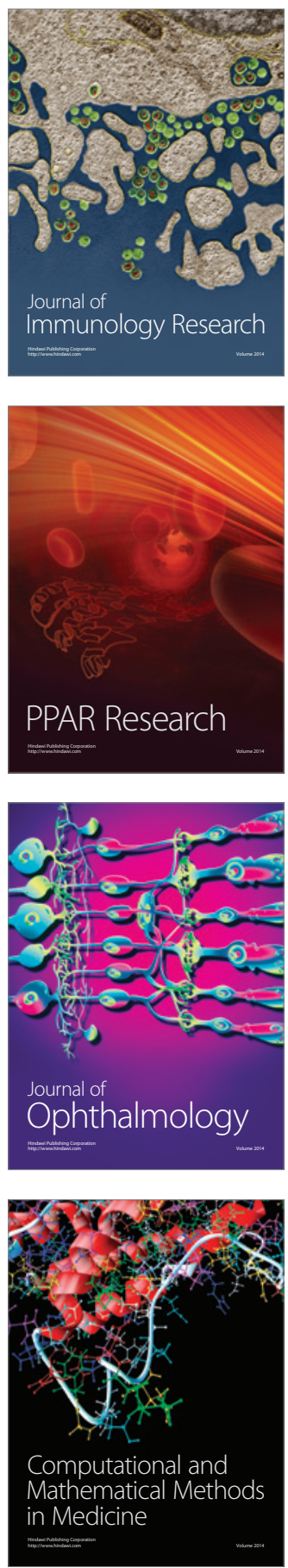

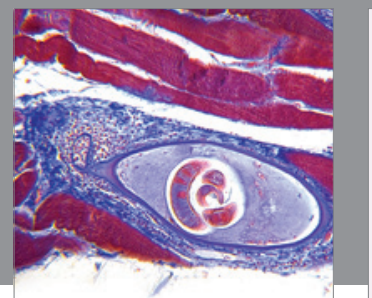

Gastroenterology

Research and Practice
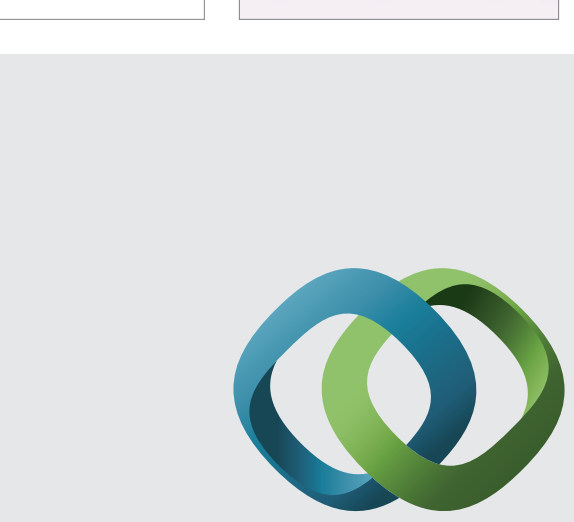

\section{Hindawi}

Submit your manuscripts at

http://www.hindawi.com
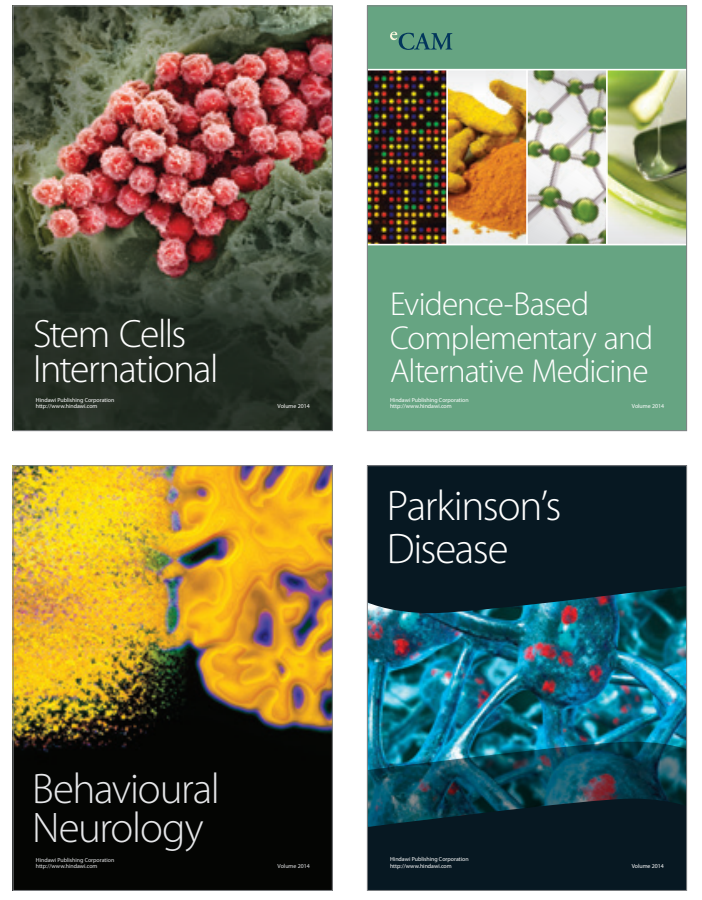
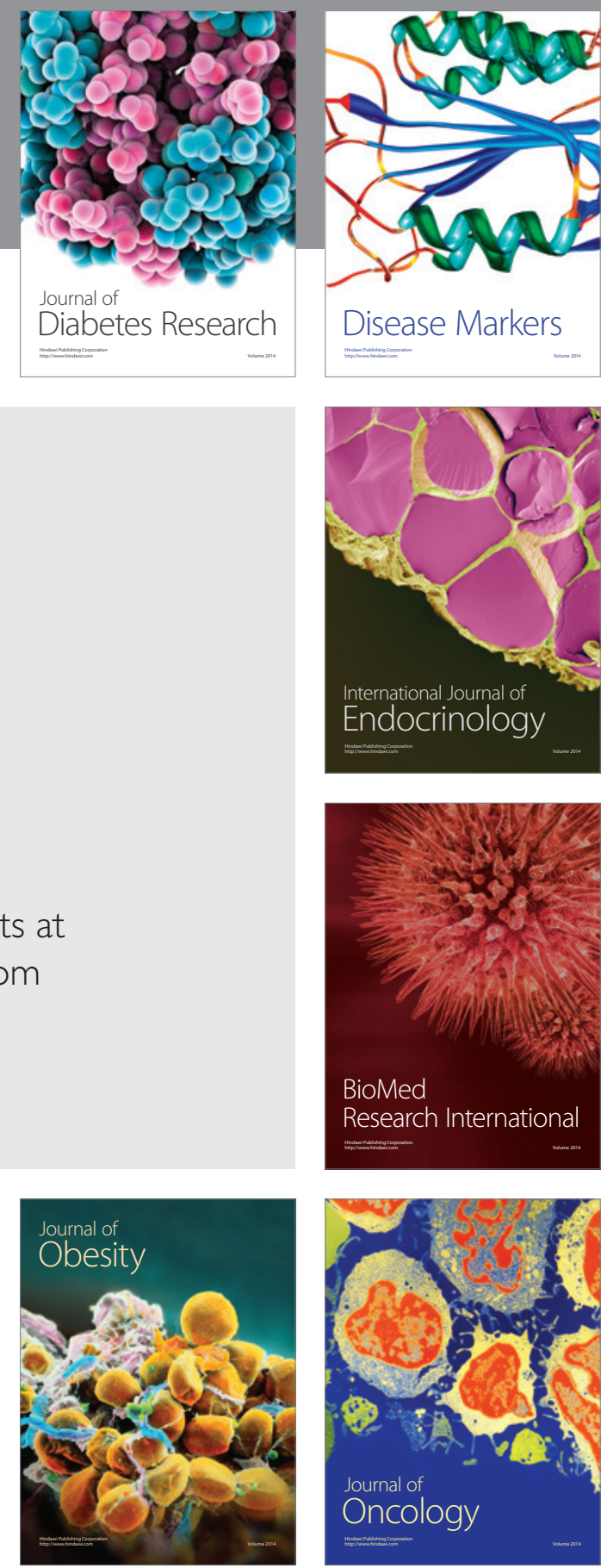

Disease Markers
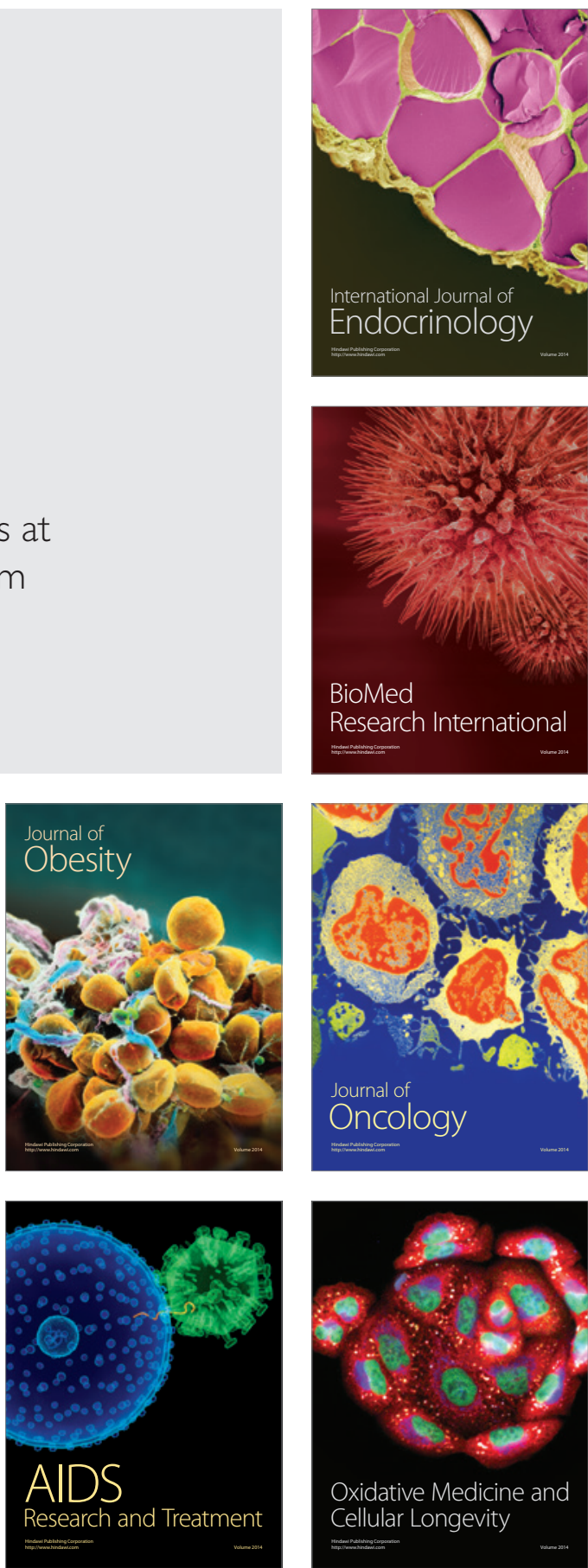